\title{
OPTIMAL PRICING FOR INSTALLBASE BUSINESS OF A MONOPOLIST CONSIDERING THREE REPRESENTATIVE CONSUMER SEGMENTS
}

\author{
Hiroaki Sandoh \\ Osaka University
}

\author{
Takeshi Koide \\ Konan University
}

(Received September 3, 2009; Revised February 19, 2010)

\begin{abstract}
The present study proposes a theoretical model to discuss an optimal pricing strategy for the installbase business under monopoly. The installbase business considered here is such that a manufacturer deals in a system along with its replaceable parts as expendable supplies which are indispensable for the system to provide its services. The proposed model introduces three representative types of consumer segments : (1) a segment of power users who show rational purchase behaviors and use the system very frequently, (2) a segment of rational normal users who also have rationality in purchase behaviors but do not use the system very frequently, and (3) a segment of myopic normal users who are stimulated to purchase a system only by the system price and do not use it very frequently. Under the proposed model, it is suggested that the manufacturer should not target the power users only but should attract myopic normal users as well to be successful in the business.
\end{abstract}

Keywords: Economics, installbase business, expendable supplies, complementary goods, consumer segments, optimal pricing

\section{Introduction}

The present study discusses an optimal pricing strategy for the installbase business under monopoly. The installbase business considered here is such that the manufacturer deals in a system along with its replaceable parts as expendable supplies (expendables in short) that are indispensable for the system to provide its services. Among several types of installbase businesses, we confine ourselves to a specific type of the installbase business, where the manufacturer sells the system at a relatively lower price and expendables at a higher price instead. Such a business can be observed in Canon in Japan which sells printers along with toner cartridges, Gillette in the USA which supplies razors with razor blade cartridges and Brita in Germany which deals in water purifiers together with water filters.

The expendables are generically explained in terms of complementary goods (complements in short) against substitute goods (substitutes in short) in economics (see, e.g., Mankiw [5]), since the markdown on the system will increase the demand of expendables. Most of studies on complements (occasionally along with substitutes) deal with problems in the context of duopolistic or oligopolistic competition between firms, that is, when firm $\mathcal{A}$ deals in a specific kind of goods, its competitor(s) sells the complements (together with substitutes) against firm $\mathcal{A}$ (see, e.g., Bulow et al. [1], Willson [9], Vives [8], in recent years, Dubey et al. [2] and Karp et al. [3]).

In this study, however, we consider a monopolist who deals in both the system and expendables, and discuss an optimal pricing for them from the viewpoint of revenue management [7], which is one of the most successful application areas of operations research. 
Assuming that one or a few expendables are initially attached to each individual system so that the consumer can use it promptly, we here introduce three typical types of consumer segments: (1) a segment of power users, (2) a segment of rational normal users and (3) a segment of myopic normal users. Both power users and rational normal ones show rational purchase behaviors as usually postulated in economics. However, myopic users do not refer to the price of expendables but to the system price only when they purchase a system. They notice the expendable price at the moment of purchasing some additional expendables immediately after they consume all the initially attached ones. The concept of myopic users has been introduced in this study since we can see such users commonly in the actual environments and above all they play an important role as will be described later.

The consumer segmentation in the above has originally been proposed by Sandoh and Koide [6]. A similar concept has also been considered by Khouja et al. [4] in the context of pricing with rebates for ordinary goods.

A theoretical model is developed to discuss an optimal pricing strategy for the system and expendables under monopoly and explored are the conditions under which the installbase business becomes successful.

\section{Notations and Assumptions}

We make the following assumptions:

(1) We consider a monopoly.

(2) Each individual system is initially attached $k$ free expendables so that the consumer can use the system promptly after they purchase it, where $k$ is a suitable positive integer.

(3) Consumers are classified into two representative segments: (1) power users and (2) normal ones on the basis of the type of system usage, e.g., the frequency of system usage and/or the system operation time. The power user is assumed to show rational purchase behaviors as is postulated in economics, but the normal user is not necessarily rational as explained in the next assumption.

(4) The normal users are further classified into two typical segments: (2-a) rational normal users who show rational purchase behaviors and (2-b) myopic normal users who are not rational in the sense that they do not refer to the price of expendables but are interested in the system price only when they purchase a system. The myopic normal users would notice the expendable price in case they become interested in purchasing some additional expendables after they consume all the initially attached ones. In this study, we generically express the power user and the rational normal user as the rational consumer, while the myopic normal users are simply called the myopic consumers.

(5) A power user has WTP (Willingness To Pay) denoted by $r_{1} \mu_{1}$ for the system use, where $r_{1}$ and $\mu_{1}$ respectively signify WTP per expendable and the mean number of expendables to be consumed until the system expires. On the other hand, a normal user (a rational normal user and a myopic normal one) has WTP given by $r_{2} \mu_{2}$ for the system use, where $r_{2}$ and $\mu_{2}$ have the same meaning as for the power user. It is postulated that $0<r_{2}<r_{1}$ and $0<\mu_{2}<\mu_{1}$.

(6) The segment sizes of the power users, the rational normal users and the myopic ones in the market are denoted by $N_{1}, N_{2}$ and $N_{3}$, respectively, with $0<N_{i}<\infty$ for $i=1,2,3$.

(7) The raw price of a system is denoted by $a\left(q_{1}\right)$, while that of an expendable is expressed by $b\left(q_{2}\right)$, where $q_{1}$ and $q_{2}$ respectively signify the volume of the systems and the expendables to be produced or to be supplied in the market. These raw prices $a\left(q_{1}\right)$ and $b\left(q_{2}\right)$ are, 
in economics, referred to as the average total costs for the system and the expendables, respectively.

(8) Let us denote, by $p_{1}(>0)$, the selling price of a system, and by $p_{2}(>0)$, the selling price of an expendable. Note that the design variables are the system price $p_{1}$ and the price $p_{2}$ of an expendable for the manufacturer.

(9) The number $k$ of free expendables initially attached to each individual system satisfies $k<\mu_{2}$ since if we have $k \geq \mu_{2}$, the normal users would not be interested in additional expendables.

\section{Optimal Response of Market}

\subsection{Consumer's utility}

Let us define the structure of utility of a rational consumer by

$$
u_{i}\left(p_{1}, p_{2}\right)=r_{i} \mu_{i}-p_{1}-\left(\mu_{i}-k\right) p_{2}, \quad i=1,2
$$

where $i=1$ and $i=2$ correspond to a power user and a rational normal one, respectively.

If $u_{i}\left(p_{1}, p_{2}\right)>0$, a rational consumer will purchase a system. After all the expendables initially attached to the system are consumed, a rational consumer will buy additional expendables for the continued use of the system. In the case of $u_{i}\left(p_{1}, p_{2}\right)<0$, on the contrary, it is needless to say that a rational consumer would buy neither the system nor expendables. When we have $u_{i}\left(p_{1}, p_{2}\right)=0$, purchasing a system along with additional expendables is indifferent to not purchasing them for these consumers, but this study regards such a case as not purchasing them since it expresses a worse situation for the manufacturer.

As for a myopic consumer, on the other hand, this study regulates his/her purchase behavior as follows:

(I) The myopic consumer makes a decision whether to purchase a system or not only if $u_{1}\left(p_{1}, p_{2}\right)>0$. In the case of $u_{1}\left(p_{1}, p_{2}\right) \leq 0$, the myopic consumer would buy neither a system nor expendables. This indicates that the system would not attract the myopic users unless the power user should buy one.

(II) The decision making by the myopic consumer consists of the following two phases;

i) In the first phase, the myopic consumer focuses on the immediate utility given by

$$
u_{3}^{(I)}\left(p_{1}, p_{2}\right)=r_{2} \mu_{2}-p_{1}
$$

If $u_{3}^{(I)}\left(p_{1}, p_{2}\right)>0$, the myopic consumer will at least purchase a system, and otherwise he/she would never buy the system. Equation (3.2) signifies that the myopic consumer is not concerned about the expendable price in purchasing the system since $k$ expendables are initially attached to each individual system.

ii) After the myopic consumer consumes all the $k$ expendables initially attached to the system, he/she will notice their price $p_{2}$ to make the second decision whether or not he/she should purchase additional expendables for the continued use of the system. In this phase, the myopic consumer might realize his/her net utility is actually given by

$$
u_{3}\left(p_{1}, p_{2}\right)=r_{2} \mu_{2}-p_{1}-\left(\mu_{2}-k\right) p_{2}
$$

and occasionally $u_{3}\left(p_{1}, p_{2}\right) \leq 0$. Nevertheless, even if the actual net utility of the myopic consumer is found to be negative, he/she will regard the initial cost 
spent in buying the system as a sunk cost to introduce the second criterion given by

$$
u_{3}^{(I I)}\left(p_{1}, p_{2}\right)=r_{2}\left(\mu_{2}-k\right)-p_{2}\left(\mu_{2}-k\right) .
$$

If $u_{3}^{(I I)}\left(p_{1}, p_{2}\right)>0$, i.e., $r_{2}>p_{2}$, the myopic consumer will buy expendables additionally for the continued use of the system. Equation (3.4) does not involve the system price because it is regarded as a sunk cost in this phase.

As we have seen in the above, the decision making in the second phase sounds to be rational, but that in the first phase implies the limited ability of the myopic consumer to process the future information. The information for consumers is seemingly simple, but the rational decision making requires the information on the price of an expendable together with the mean number of expendables to be consumed over a long time span as well as the system price. These messy factors may possibly confuse the myopic consumer to prevent them from behaving rationally. In addition, it is interesting that such purchase behaviors can frequently be observed in the real circumstances.

\subsection{Additional assumptions}

The previous subsection has described the structures of consumer's utilities. This subsection introduces some additional assumptions, letting $N$ and $M$ be defined by

$$
\begin{aligned}
& N=N_{1}+N_{2}+N_{3}, \\
& M=N_{1} \mu_{1}+\left(N_{2}+N_{3}\right) \mu_{2} .
\end{aligned}
$$

In Equation (3.5), $N$ expresses the entire population size or the aggregate segment size of the consumers, namely the maximum demand for the systems. Likewise, $M$ in Equation (3.6) expresses the maximum demand for the expendables.

We here focus on the average total production cost $a\left(q_{1}\right)$ of the system. The general economical analysis assumes the domain for $q_{1}$ is $(0, \infty)$. In this study, however, we introduce $N_{1} \leq q_{1} \leq N<\infty$ as the domain for $q_{1}$ considering actual business environments. This indicates that there exists $q_{1}^{*}$ that maximizes $a\left(q_{1}\right)$. As for the average total production cost $b\left(q_{2}\right)$ of expendables, we introduce $N_{1} \mu_{1} \leq q_{2} \leq M<\infty$, and the similar considerations to the above will result in the existence of $q_{2}^{*}$ which maximizes $b\left(q_{2}\right)$. It should be noted that $N_{1}$ and $N_{1} \mu_{1}$ are the lower bounds for $q_{1}$ and $q_{2}$, respectively, and the reason for this will become clear later.

Based on these preliminaries, we introduce two additional assumptions as follows:

(10) $r_{i} \mu_{i}-a\left(q_{1}^{*}\right)-\mu_{i} b\left(q_{2}^{*}\right)>0, \quad i=1,2$,

(11) $\frac{r_{1} \mu_{1}}{\mu_{1}-k}>\frac{r_{2} \mu_{2}}{\mu_{2}-k}$.

Assumption (10) signifies that if the consumers can purchase the system and additional expendables at each raw price $a\left(q_{1}\right)$ and $b\left(q_{2}\right)$, respectively, they would obtain positive utility from the system without any expendables even though $a\left(q_{1}\right)$ and $b\left(q_{2}\right)$ should be set to each highest value $a\left(q_{1}^{*}\right)$ and $b\left(q_{2}^{*}\right)$, respectively. Assumption (11) means that WTP per additional expendable of a power user is larger than that of a normal user.

\subsection{Optimal response}

On the basis of the utility function we have formulated for each type of consumers, the optimal response of the market becomes as follows:

Let us define $\Omega_{i}(i=0,1,2)$ and $\Omega_{3(j)}(j=1,2)$ as follows:

$$
\Omega_{0}=\left\{\left(p_{1}, p_{2}\right) \mid u_{i}\left(p_{1}, p_{2}\right) \leq 0(i=1,2), u_{3}^{(I)}\left(p_{1}, p_{2}\right) \leq 0\right\}
$$




$$
\begin{aligned}
\Omega_{1} & =\left\{\left(p_{1}, p_{2}\right) \mid u_{1}\left(p_{1}, p_{2}\right)>0, u_{2}\left(p_{1}, p_{2}\right) \leq 0, u_{3}^{(I)}\left(p_{1}, p_{2}\right) \leq 0\right\} \\
\Omega_{2} & =\left\{\left(p_{1}, p_{2}\right) \mid u_{2}\left(p_{1}, p_{2}\right)>0\right\} \\
\Omega_{3(1)} & =\left\{\left(p_{1}, p_{2}\right) \mid u_{1}\left(p_{1}, p_{2}\right)>0, u_{2}\left(p_{1}, p_{2}\right) \leq 0, u_{3}^{(I)}\left(p_{1}, p_{2}\right)>0, u_{3}^{(I I)}\left(p_{1}, p_{2}\right) \leq 0\right\} \\
\Omega_{3(2)} & =\left\{\left(p_{1}, p_{2}\right) \mid u_{1}\left(p_{1}, p_{2}\right)>0, u_{2}\left(p_{1}, p_{2}\right) \leq 0, u_{3}^{(I)}\left(p_{1}, p_{2}\right)>0, u_{3}^{(I I)}\left(p_{1}, p_{2}\right)>0\right\}
\end{aligned}
$$

If $\left(p_{1}, p_{2}\right) \in \Omega_{0}$, none of the rational and the myopic consumer would purchase the system, and consequently none of them would buy expendables. This is because both the system and the expendable are very expensive for every type of consumers. In the case of $\left(p_{1}, p_{2}\right) \in \Omega_{1}$, only the power user would purchase the system and additional expendables when needed for the continued use of the system. When we have $\left(p_{1}, p_{2}\right) \in \Omega_{2}$, all the types of consumers would buy the system along with expendables as necessary since both the system and the expendable have reasonable prices.

If the manufacturer plots $\left(p_{1}, p_{2}\right) \in \Omega_{3(1)}$, the power user would purchase the system and sooner or later additional expendables, but the rational normal user would buy neither the system nor expendables. As for the myopic normal user, however, he/she would purchase the system since the condition of the first phase decision making is satisfied, but would never pay for additional expendables because the condition of the second phase decision making is not satisfied due to the high price of the expendables. When the manufacturer schemes $\left(p_{1}, p_{2}\right) \in \Omega_{3(2)}$, likewise, the power user would purchase the system and expendables when necessary, and the rational normal user would buy neither the system nor expendables. However, the myopic normal user would show the same purchase behaviors as those of the power user, that is, he/she would purchase the system and additional expendables when needed for the continued use of the system.

The market's optimal response discussed in the above can be depicted in two different figures depending on whether a relationship, $r_{1} \mu_{1}<r_{2}\left(\mu_{1}+\mu_{2}-k\right)$, holds or not.

Figure 1 shows the five regions, $\Omega_{i}(i=0,1,2)$ and $\Omega_{3(j)}(j=1,2)$, on the $\left(p_{1}, p_{2}\right)$ plane in the case of $r_{1} \mu_{1}<r_{2}\left(\mu_{1}+\mu_{2}-k\right)$. Besides, the signification of Assumption (11) is represented on the vertical axis in Figure 1. Figure 2 reveals the five regions on the $\left(p_{1}, p_{2}\right)$ plane when we have $r_{1} \mu_{1} \geq r_{2}\left(\mu_{1}+\mu_{2}-k\right)$. Assumption (11) is reflected on the vertical axis also in Figure 2. It is to be noted that the difference between Figures 1 and 2 is observed simply in the location of the horizontal line given by $p_{2}=r_{2}$, but this difference significantly affects the manufacturer's optimal pricing strategy.

Table 1 summarizes the strict definitions of these five regions for the case of $r_{1} \mu_{1}<$ $r_{2}\left(\mu_{1}+\mu_{2}-k\right)$ and $r_{1} \mu_{1} \geq r_{2}\left(\mu_{1}+\mu_{2}-k\right)$.

\section{Manufacturer's Profit}

This section derives the manufacturer's profit on the basis of the optimal response of the market we have investigated in the previous section.

\subsection{In the case of $r_{1} \mu_{1}<r_{2}\left(\mu_{1}+\mu_{2}-k\right)$}

In this case, the optimal response of the market was described in Figure 1 in Section 3.3. The details of the manufacturer's profit are discussed in the following:

When the manufacturer sets the prices $\left(p_{1}, p_{2}\right)$ of the system and the expendable in $\left(p_{1}, p_{2}\right) \in \Omega_{0}$, no consumer would purchase the system, accordingly it is needless to say that no consumer would buy the expendables. This indicates the manufacturer's profit is given 


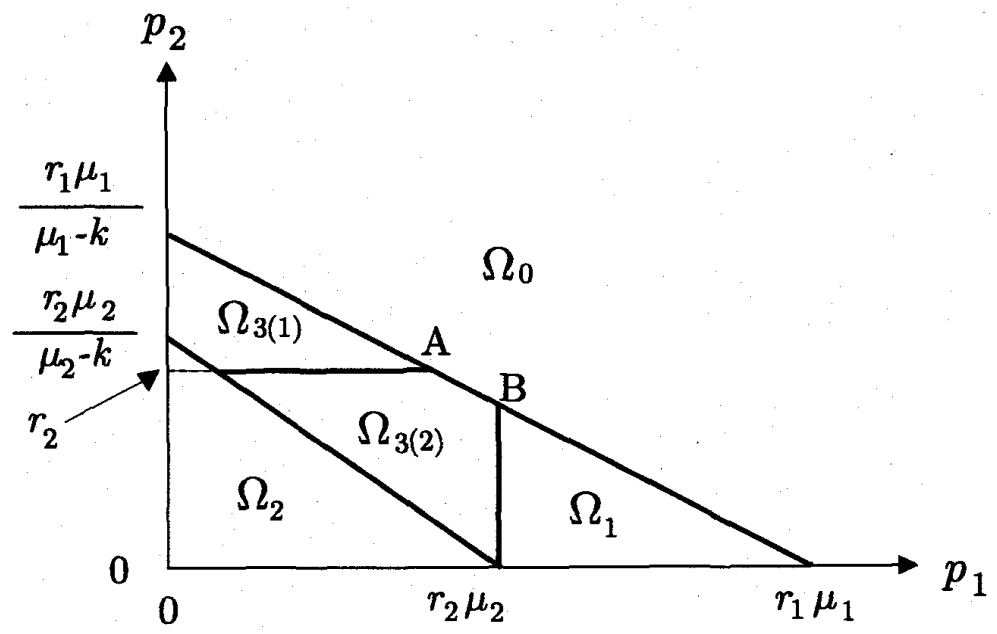

Figure 1: Optimal response in the case of $r_{1} \mu_{1}<r_{2}\left(\mu_{1}+\mu_{2}-k\right)$

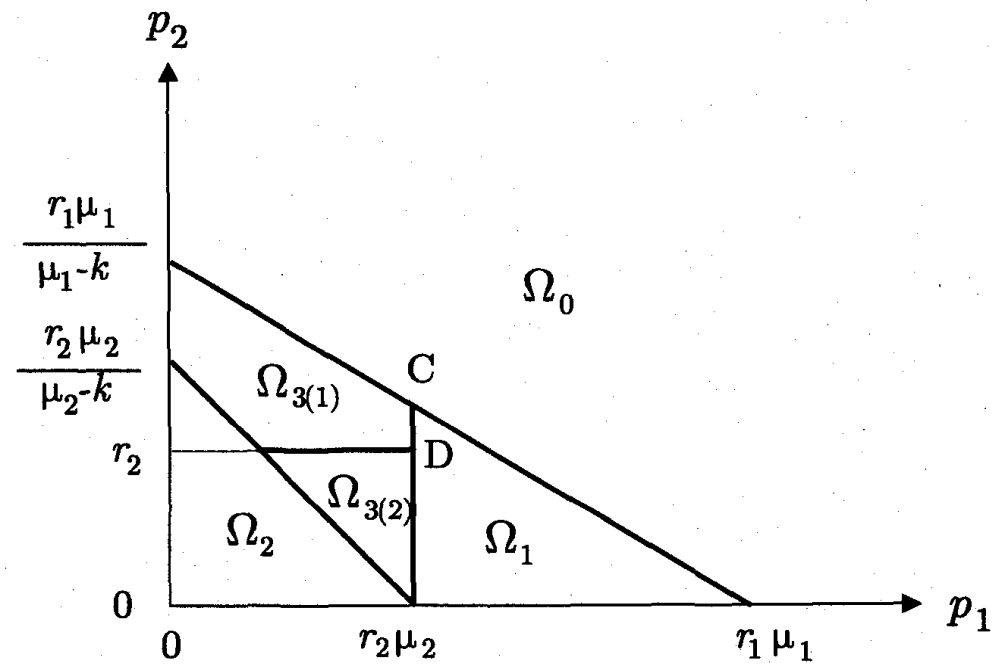

Figure 2: Optimal response in the case of $r_{1} \mu_{1} \geq r_{2}\left(\mu_{1}+\mu_{2}-k\right)$

Table 1: Strict definitions of optimal response

\begin{tabular}{l}
\hline$r_{1} \mu_{1}<r_{2}\left(\mu_{1}+\mu_{2}-k\right)$ \\
\hline$\Omega_{0}=\left\{\left(p_{1}, p_{2}\right) \mid p_{1}>0, p_{2}>0, p_{1}+\left(\mu_{1}-k\right) p_{2} \geq r_{1} \mu_{1}\right\}$ \\
$\Omega_{1}=\left\{\left(p_{1}, p_{2}\right) \mid p_{1} \geq r_{2} \mu_{2}, p_{2}>0, p_{1}+\left(\mu_{1}-k\right) p_{2}<r_{1} \mu_{1}\right\}$ \\
$\Omega_{2}=\left\{\left(p_{1}, p_{2}\right) \mid p_{1}>0, p_{2}>0, p_{1}+\left(\mu_{2}-k\right) p_{2}<r_{2} \mu_{2}\right\}$ \\
$\Omega_{3(1)}=\left\{\left(p_{1}, p_{2}\right) \mid p_{1}>0, p_{2} \geq r_{2}, p_{1}+\left(\mu_{1}-k\right) p_{2}<r_{1} \mu_{1}, p_{1}+\left(\mu_{2}-k\right) p_{2} \geq r_{2} \mu_{2}\right\}$ \\
$\Omega_{3(2)}=\left\{\left(p_{1}, p_{2}\right) \mid p_{1}<r_{2} \mu_{2}, p_{2}<r_{2}, p_{1}+\left(\mu_{1}-k\right) p_{2}<r_{1} \mu_{1}, p_{1}+\left(\mu_{2}-k\right) p_{2} \geq r_{2} \mu_{2}\right\}$ \\
\hline$r_{1} \mu_{1} \geq r_{2}\left(\mu_{1}+\mu_{2}-k\right)$ \\
\hline$\Omega_{0}=\left\{\left(p_{1}, p_{2}\right) \mid p_{1}>0, p_{2}>0, p_{1}+\left(\mu_{1}-k\right) p_{2} \geq r_{1} \mu_{1}\right\}$ \\
$\Omega_{1}=\left\{\left(p_{1}, p_{2}\right) \mid p_{1} \geq r_{2} \mu_{2}, p_{2}>0, p_{1}+\left(\mu_{1}-k\right) p_{2}<r_{1} \mu_{1}\right\}$ \\
$\Omega_{2}=\left\{\left(p_{1}, p_{2}\right) \mid p_{1}>0, p_{2}>0, p_{1}+\left(\mu_{2}-k\right) p_{2}<r_{2} \mu_{2}\right\}$ \\
$\Omega_{3(1)}=\left\{\left(p_{1}, p_{2}\right) \mid 0<p_{1}<r_{2} \mu_{2}, p_{2} \geq r_{2}, p_{1}+\left(\mu_{1}-k\right) p_{2}<r_{1} \mu_{1}, p_{1}+\left(\mu_{2}-k\right) p_{2} \geq r_{2} \mu_{2}\right\}$ \\
$\Omega_{3(2)}=\left\{\left(p_{1}, p_{2}\right) \mid p_{1}<r_{2} \mu_{2}, p_{2}<r_{2}, p_{1}+\left(\mu_{2}-k\right) p_{2} \geq r_{2} \mu_{2}\right\}$ \\
\hline
\end{tabular}


by

$$
\pi_{0}=0
$$

Hence, in this case, the utility of a power, a rational normal and a myopic normal user are given by

$$
u_{i}^{*}=0, i=1,2,3 .
$$

If the manufacturer adopts $\left(p_{1}, p_{2}\right) \in \Omega_{1}$, only power users would buy the system and the expendables when needed, and thus the manufacturer's profit becomes

$$
\begin{aligned}
\pi_{1}\left(p_{1}, p_{2}\right) & =N_{1}\left\{p_{1}-a\left(N_{1}\right)-k b\left(N_{1} \mu_{1}\right)+\left(\mu_{1}-k\right)\left[p_{2}-b\left(N_{1} \mu_{1}\right)\right]\right\} \\
& =N_{1}\left[p_{1}+p_{2}\left(\mu_{1}-k\right)-a\left(N_{1}\right)-\mu_{1} b\left(N_{1} \mu_{1}\right)\right] .
\end{aligned}
$$

The manufacturer would seek a local maximum of his/her profit in Equation (4.3) within $\Omega_{1}$ if there exists one, or would search for its local supremum noting that $\Omega_{1}$ does not involve the boundary between $\Omega_{0}$ and $\Omega_{1}$. Easy is such local optimization of $\pi_{1}\left(p_{1}, p_{2}\right)$ in reference to $\left(p_{1}, p_{2}\right)$ since the problem can be formulated as a simple linear programming problem, and we will notice that the manufacturer does not have any maximum of the profit but has its supremum instead.

Let $\left(p_{1}^{*}, p_{2}^{*}\right)$ denote an arbitrary point on the boundary between $\Omega_{1}$ and $\Omega_{0}$ for $r_{2} \mu_{2} \leq$ $p_{1}<r_{1} \mu_{1}$, then manufacturer's local optimal pricing strategy is given by letting $p_{1} \rightarrow p_{1}^{*}-0$ and $p_{2} \rightarrow p_{2}^{*}-0$, and the supremum of the manufacturer's profit becomes

$$
\pi_{1}^{*}=\lim _{\left(p_{1}, p_{2}\right) \rightarrow\left(p_{1}^{*}-0, p_{2}^{*}-0\right)} \pi_{1}\left(p_{1}, p_{2}\right)=N_{1}\left[r_{1} \mu_{1}-a\left(N_{1}\right)-\mu_{1} b\left(N_{1} \mu_{1}\right)\right] .
$$

The power user's utility in this case becomes

$$
u_{1}^{*}=\lim _{\left(p_{1}, p_{2}\right) \rightarrow\left(p_{1}^{*}-0, p_{2}^{*}-0\right)} u_{1}\left(p_{1}, p_{2}\right)=0 .
$$

Since rational normal and myopic normal users would purchase neither the system nor the expendables, their utilities also become $u_{2}^{*}=u_{3}^{*}=0$.

When the manufacturer sets $\left(p_{1}, p_{2}\right)$ in $\Omega_{2}$, all the types of consumers would purchase the system and expendables if necessary as we have seen in the previous section, accordingly the manufacturer's profit in this case is given by

$\pi_{2}\left(p_{1}, p_{2}\right)=N_{1}\left[p_{1}+p_{2}\left(\mu_{1}-k\right)-a(N)-\mu_{1} b(M)\right]+\left(N_{2}+N_{3}\right)\left[p_{1}+p_{2}\left(\mu_{2}-k\right)-a(N)-\mu_{2} b(M)\right]$.

It is a simple linear programming problem to locally maximize $\pi_{2}\left(p_{1}, p_{2}\right)$ in Equation (4.6) with respect to $\left(p_{1}, p_{2}\right)$ in $\Omega_{2}$ or to obtain its local supremum. The result of such local optimization is to let $p_{1} \rightarrow p_{1}^{*}+0$ and $p_{2} \rightarrow p_{2}^{*}-0$, where $p_{1}^{*}$ and $p_{2}^{*}$ are given by

$$
p_{1}^{*}=0, \quad p_{2}^{*}=\frac{r_{2} \mu_{2}}{\mu_{2}-k} .
$$

It to be noted in the above that the right-hand limit of the system price is zero, though the left-hand limit of the expendable price is its highest value within $\Omega_{2}$. by

These observations reveal that the local supremum of the manufacturer's profit is given

$$
\pi_{2}^{*}=N_{1}\left[r_{2} \mu_{2} \frac{\mu_{1}-k}{\mu_{2}-k}-a(N)-\mu_{1} b(M)\right]+\left(N_{2}+N_{3}\right)\left[r_{2} \mu_{2}-a(N)-\mu_{2} b(M)\right]
$$


and the utility of each type of the consumer becomes

$$
u_{1}^{*}=r_{1} \mu_{1}-\frac{r_{2} \mu_{2}\left(\mu_{1}-k\right)}{\mu_{2}-k}, \quad u_{2}^{*}=u_{3}^{*}=0
$$

Since we have $u_{1}^{*}>0$, only the power users can obtain positive utility.

If the manufacturer plots $\left(p_{1}, p_{2}\right) \in \Omega_{3(1)}$, the rational normal user is not interested in purchasing a system, but the power user would purchase both the system and additional expendables as the need arises. The myopic normal user would buy a system, but would not pay for additional expendables owing to their high price. It follows that the manufacture's profit is given by

$$
\begin{aligned}
\pi_{3(1)}\left(p_{1}, p_{2}\right)= & N_{1}\left[p_{1}+p_{2}\left(\mu_{1}-k\right)-a\left(N_{1}+N_{3}\right)-\mu_{1} b\left(N_{1} \mu_{1}+N_{3} k\right)\right] \\
& +N_{3}\left[p_{1}-a\left(N_{1}+N_{3}\right)-k b\left(N_{1} \mu_{1}+N_{3} k\right)\right]
\end{aligned}
$$

The manufacturer's locally optimal pricing strategy in this region $\Omega_{3(1)}$, which is easily obtained by solving a simple linear programming problem, is to let $p_{1} \rightarrow p_{1}^{*}-0$ and $p_{2} \rightarrow$ $p_{2}^{*}-0$, where

$$
p_{1}^{*}=r_{1} \mu_{1}-r_{2}\left(\mu_{1}-k\right), \quad p_{2}^{*}=r_{2},
$$

which is represented by point " $\mathrm{A}$ " in Figure 1. The local supremum of the manufacturer's profit is, at point "A", given by

$$
\begin{aligned}
\pi_{3(1) a}^{*}= & N_{1}\left[r_{1} \mu_{1}-a\left(N_{1}+N_{3}\right)-\mu_{1} b\left(N_{1} \mu_{1}+N_{3} k\right)\right] \\
& +N_{3}\left[r_{1} \mu_{1}-r_{2}\left(\mu_{1}-k\right)-a\left(N_{1}+N_{3}\right)-k b\left(N_{1} \mu_{1}+N_{3} k\right)\right]
\end{aligned}
$$

and the utility of each type of consumers becomes

$$
u_{1}^{*}=u_{2}^{*}=0, u_{3}^{*}=-\left(r_{1}-r_{2}\right) \mu_{1}, u_{3}^{(I) *}=r_{2}\left(\mu_{1}+\mu_{2}-k\right)-r_{1} \mu_{1} .
$$

In the above, we have $u_{3}^{*}<0$ though $u_{3}^{(I) *}>0$. This reveals that in purchasing a system, the myopic consumer deems his/her utility positive since the utility in the first phase $u_{3}^{(I) *}$ takes a positive value although his/her actual net utility $u_{3}^{*}$ considering the cost for additional expendables turns out to be negative. In the second phase decision making, however, the myopic consumer would not pay for additional expendables on account of its high price.

On the other hand, when the manufacturer schemes a pricing strategy $\left(p_{1}, p_{2}\right) \in \Omega_{3(2)}$, the rational normal user would buy neither the system nor expendables. However, both the power user and the myopic normal user would purchase the system and additional expendables after they consume all the initially attached expendables. Consequently, the manufacturer's profit is given by

$$
\begin{aligned}
\pi_{3(2)}\left(p_{1}, p_{2}\right)= & N_{1}\left[p_{1}+p_{2}\left(\mu_{1}-k\right)-a\left(N_{1}+N_{3}\right)-\mu_{1} b\left(N_{1} \mu_{1}+N_{3} \mu_{2}\right)\right] \\
& +N_{3}\left[p_{1}+p_{2}\left(\mu_{2}-k\right)-a\left(N_{1}+N_{3}\right)-\mu_{2} b\left(N_{1} \mu_{1}+N_{3} \mu_{2}\right)\right]
\end{aligned}
$$

and the supremum of $\pi_{3(2)}\left(p_{1}, p_{2}\right)$ locally in $\Omega_{3(2)}$ is easily obtained by letting $p_{1} \rightarrow p_{1}^{*}-0$ and $p_{2} \rightarrow p_{2}^{*}-0$, where

$$
p_{1}^{*}=r_{2} \mu_{2}, \quad p_{2}^{*}=\frac{r_{1} \mu_{1}-r_{2} \mu_{2}}{\mu_{1}-k},
$$

which is represented by point " $\mathrm{B}$ " in Figure 1. 
The local optimal pricing in the above provides the manufacturer with the supremum of his/her profit expressed as

$$
\begin{aligned}
\pi_{3(2) a}^{*}= & N_{1}\left[r_{1} \mu_{1}-a\left(N_{1}+N_{3}\right)-\mu_{1} b\left(N_{1} \mu_{1}+N_{3} \mu_{2}\right)\right] \\
& +N_{3}\left[r_{2} \mu_{2}+\frac{\left(r_{1} \mu_{1}-r_{2} \mu_{2}\right)\left(\mu_{2}-k\right)}{\mu_{1}-k}-a\left(N_{1}+N_{3}\right)-\mu_{2} b\left(N_{1} \mu_{1}+N_{3} \mu_{2}\right)\right]
\end{aligned}
$$

and the utility of each type of consumers is given by

$$
\left.\begin{array}{ll}
u_{1}^{*}=u_{2}^{*}=0, & u_{3}^{*}=-\frac{\left(r_{1} \mu_{1}-r_{2} \mu_{2}\right)\left(\mu_{2}-k\right)}{\mu_{1}-k} \\
u_{3}^{(I) *}=0, & u_{3}^{(I I) *}=r_{2}\left(\mu_{2}-k\right)-\frac{\left(r_{1} \mu_{1}-r_{2} \mu_{2}\right)\left(\mu_{2}-k\right)}{\mu_{1}-k} .
\end{array}\right\}
$$

Equation (4.17) indicates that $u_{3}^{*}<0$ and $u_{3}^{(I I) *}>0$; in other words, that only the myopic normal user can obtain positive utility in the second phase decision making although his/her actual net utility is zero. This is because the myopic normal user would regard the initial cost in purchasing the system as a sunk cost as mentioned in Section 3.1.

\subsection{In the case of $r_{1} \mu_{1} \geq r_{2}\left(\mu_{1}+\mu_{2}-k\right)$}

In case we have $r_{1} \mu_{1} \geq r_{2}\left(\mu_{1}+\mu_{2}-k\right)$, the optimal response of the market was depicted in Figure 2. The analyses for the cases of $\left(p_{1}, p_{2}\right) \in \Omega_{i}$ for $i=0,1,2$ yield quite the same results as those we have seen for the case of $r_{1} \mu_{1}<r_{2}\left(\mu_{1}+\mu_{2}-k\right)$. For this reason, this subsection confines itself to the cases of $\left(p_{1}, p_{2}\right) \in \Omega_{3(1)}$ and $\left(p_{1}, p_{2}\right) \in \Omega_{3(2)}$ in the following.

If the manufacturer schemes $\left(p_{1}, p_{2}\right) \in \Omega_{3(1)}$, his/her profit is given by Equation (4.10), and the locally optimal pricing strategy is given by letting $p_{1} \rightarrow p_{1}^{*}-0$ and $p_{2} \rightarrow p_{2}^{*}-0$, where

$$
p_{1}^{*}=r_{2} \mu_{2}, \quad p_{2}^{*}=\frac{r_{1} \mu_{1}-r_{2} \mu_{2}}{\mu_{1}-k}
$$

which corresponds to point " $\mathrm{C}$ " in Figure 2. Then, point "C" in Figure 2 provides the local supremum of the manufacturer's profit expressed as

$$
\begin{aligned}
\pi_{3(1) b}^{*}= & N_{1}\left[r_{1} \mu_{1}-a\left(N_{1}+N_{3}\right)-\mu_{1} b\left(N_{1} \mu_{1}+N_{3} k\right)\right] \\
& +N_{3}\left[r_{2} \mu_{2}-a\left(N_{1}+N_{3}\right)-k b\left(N_{1} \mu_{1}+N_{3} k\right)\right]
\end{aligned}
$$

and the consumer's utility to be realized becomes

$$
u_{1}^{*}=u_{2}^{*}=0, \quad u_{3}^{*}=-\frac{\left(r_{1} \mu_{1}-r_{2} \mu_{2}\right)\left(\mu_{2}-k\right)}{\mu_{1}-k}, \quad u_{3}^{(I) *}=0 .
$$

In the above equation, the myopic consumer has $u_{3}^{*}<0$, the negative actual net utility in spite of the non-negative utility in the first phase decision making.

On the other hand, if the manufacturer plots $\left(p_{1}, p_{2}\right)$ in $\Omega_{3(2)}$, the manufacturer's profit is given by Equation (4.14), and the locally optimal pricing strategy in this case is easily found to be letting $p_{1} \rightarrow p_{1}^{*}-0$ together with $p_{2} \rightarrow p_{2}^{*}-0$, where

$$
p_{1}^{*}=r_{2} \mu_{2}, \quad p_{2}^{*}=r_{2} .
$$

This is represented by point " $D$ " in Figure 2, which provides the local supremum of his/her profit expressed as

$$
\begin{aligned}
\pi_{3(2) b}^{*}= & N_{1}\left[r_{2} \mu_{2}+r_{2}\left(\mu_{1}-k\right)-a\left(N_{1}+N_{3}\right)-\mu_{1} b\left(N_{1} \mu_{1}+N_{3} \mu_{2}\right)\right] \\
& +N_{3}\left[r_{2} \mu_{2}+r_{2}\left(\mu_{2}-k\right)-a\left(N_{1}+N_{3}\right)-\mu_{2} b\left(N_{1} \mu_{1}+N_{3} \mu_{2}\right)\right]
\end{aligned}
$$


and each type of consumers will have

$$
\left.\begin{array}{ll}
u_{1}^{*}=r_{1} \mu_{1}-r_{2}\left(\mu_{1}+\mu_{2}-k\right), & u_{2}^{*}=0, \\
u_{3}^{*}=-r_{2}\left(\mu_{2}-k\right), & u_{3}^{(I) *}=u_{2}^{(I I) *}=0 .
\end{array}\right\}
$$

These results reveal that only the power user can have positive utility from the system use but that the myopic consumer has zero utility both in the first and the second phase decision making and negative actual net utility.

We have examined locally optimal pricing strategies for the manufacturer according to the consumer's response. The succeeding section summarizes the above results to explore a global optimal pricing strategy.

\section{Manufacturer's Optimal Pricing Strategy}

The manufacturer's global optimal pricing strategy can be summarized in the following manner:

(1) If we have $\max \left(\pi_{1}^{*}, \pi_{2}^{*}, \pi_{3(1) j}^{*}, \pi_{3(2) j}^{*}\right)=\pi_{1}^{*}$ for $j=a, b$, the manufacturer's optimal pricing strategy becomes to let $\left(p_{1}, p_{2}\right) \rightarrow\left(p_{1}^{*}-0, p_{2}^{*}-0\right)$, where $\left(p_{1}^{*}, p_{2}^{*}\right)$ is given by an arbitrary point on the line defined by $r_{1} \mu_{1}-p_{1}-\left(\mu_{1}-k\right) p_{2}=0$ for $r_{2} \mu_{2}<p_{1}<r_{1} \mu_{1}$.

(2) When we have $\max \left(\pi_{1}^{*}, \pi_{2}^{*}, \pi_{3(1) j}^{*}, \pi_{3(2) j}^{*}\right)=\pi_{2}^{*}$ for $j=a, b$, the manufacturer's optimal pricing strategy is featured by $\left(p_{1}^{*}, p_{2}^{*}\right)=\left(0, \frac{r_{2} \mu_{2}}{\mu_{2}-k}\right)$.

(3) In the case of $r_{1} \mu_{1}<r_{2}\left(\mu_{1}+\mu_{2}-k\right)$, the optimal pricing strategy can be discussed for the following subcases;

i) If we have $\max \left(\pi_{1}^{*}, \pi_{2}^{*}, \pi_{3(1) a}^{*}, \pi_{3(2) a}^{*}\right)=\pi_{3(1) a}^{*}$, the optimal pricing strategy is characterized by $\left(p_{1}^{*}, p_{2}^{*}\right)=\left(r_{1} \mu_{1}-r_{2}\left(\mu_{1}-k\right), r_{2}\right)$.

ii) In case we have $\max \left(\pi_{1}^{*}, \pi_{2}^{*}, \pi_{3(1) a}^{*}, \pi_{3(2) a}^{*}\right)=\pi_{3(2) a}^{*},\left(p_{1}^{*}, p_{2}^{*}\right)=\left(r_{2} \mu_{2}, \frac{r_{1} \mu_{1}-r_{2} \mu_{2}}{\mu_{1}-k}\right)$ characterizes the manufacturer's optimal pricing strategy.

(4) In the case of $r_{1} \mu_{1} \geq r_{2}\left(\mu_{1}+\mu_{2}-k\right)$, the optimal strategy becomes;

i) If we have $\max \left(\pi_{1}^{*}, \pi_{2}^{*}, \pi_{3(1) b}^{*}, \pi_{3(2) b}^{*}\right)=\pi_{3(1) b}^{*}$, the optimal strategy is featured by $\left(p_{1}^{*}, p_{2}^{*}\right)=\left(r_{2} \mu_{2}, \frac{r_{1} \mu_{1}-r_{2} \mu_{2}}{\mu_{1}-k}\right)$, which coincides with that in (3)-ii) though the supremum of the manufacturer's profit is not the same.

ii) When we have $\max \left(\pi_{1}^{*}, \pi_{2}^{*}, \pi_{3(1) b}^{*}, \pi_{3(2) b}^{*}\right)=\pi_{3(2) b}^{*}$, the optimal strategy is characterized by $\left(p_{1}^{*}, p_{2}^{*}\right)=\left(r_{2} \mu_{2}, r_{2}\right)$.

In the above, we hardly encounter the situation expressed in (1), which will be explained later through two propositions. In the case of (2), however, all the types of consumers would purchase the systems and additional expendables. This result can be considered to represent a specific type of installbase business since it suggests that the manufacturer should distribute the systems to all the types of consumers nearly free of charge because of $p_{1}^{*}=0$, and instead he/she should sell the expendables at their maximum tolerable price within $\Omega_{2}$.

The results in the cases of (3)-i) and (4)-i) explain another type of installbase business. This is because they suggest that the manufacturer should ignore the rational normal users to focus on the power users and the myopic normal users only, and that the price of expendables should be set so high that the myopic normal users might not purchase expendables for the continued use of the system. In (3)-i), the price of an expendable is set nearly equal to $r_{2}$ 
which denotes WTP per expendable of the normal user, although it is set larger than $r_{2}$ in (4)-i). Pricing strategies of this kind can occasionally be observed for some products, where many consumers purchase systems, but after they consume all the expendables initially attached to individual systems, they abandon the continued use of the systems due to expensive expendables. Such a kind of products might be to fade away from the market sooner or later or is to be purchased repeatedly only by the power users after the market is saturated with the products.

Both in (3)-ii) and (4)-ii) as well, the manufacturer should target the myopic normal users as well as the power users. Besides, the manufacturer should set the system price nearly equal to $r_{2} \mu_{2}$. In other words, the manufacturer should replace the whole WTP of a normal user for the system use with a system price. However, the myopic normal users pay for additional expendables for the continued use of the systems by regarding the system price as a sunk cost. This result represents one more type of installbase business. follows:

As for relationships among $\pi_{1}^{*}, \pi_{3(1) a}^{*}$ and $\pi_{3(2) a}^{*}$, we can derive useful propositions as

Proposition 5.1

(i) If $a\left(N_{1}\right) \geq a\left(N_{1}+N_{3}\right)$ and $b\left(N_{1} \mu_{1}\right) \geq b\left(N_{1} \mu_{1}+N_{3} \mu_{2}\right)$, we have $\pi_{3(2) a}^{*} \geq \pi_{1}^{*}$.

(ii) If $b\left(N_{1} \mu_{1}+N_{3} k\right) \geq b\left(N_{1} \mu_{1}+N_{3} \mu_{2}\right)$, we have $\pi_{3(2) a}^{*} \geq \pi_{3(1) a}^{*}$.

[Proof] See Appendix A.

Proposition 5.1-(i) signifies that the manufacturer should target not only the power users but the myopic rational normal users unless the raw prices $a\left(q_{1}\right)$ and $b\left(q_{2}\right)$ increase with $q_{1}$ and $q_{2}$, respectively. On the other hand, Proposition 5.1-(ii) insists that the manufacturer can increase his/her profit by selling expendables to the myopic normal users as well unless $b\left(q_{2}\right)$ is increasing in $q_{2}$.

We can also derive the following proposition for the relationship between $\pi_{1}^{*}$ and $\pi_{3(1) b}^{*}$ :

\section{Proposition 5.2}

If $a\left(N_{1}\right) \geq a\left(N_{1}+N_{3}\right)$ and $b\left(N_{1} \mu_{1}\right) \geq b\left(N_{1} \mu_{1}+N_{3} k\right)$, we have $\pi_{3(1) b}^{*} \geq \pi_{1}^{*}$.

[Proof] See Appendix B.

Proposition 5.2 suggests that the manufacturer should not focus on the power users only but he/she should attract the myopic normal users as well if neither $a\left(q_{1}\right)$ nor $b\left(q_{2}\right)$ increases with $q_{1}$ and $q_{2}$, respectively.

These two propositions will explain why we hardly encounter (1) in the above classification.

\section{Numerical Examples}

Table 2 shows the cases to be treated in this section. Note in Table 2 that the number of expendables initially attached to each system is $k=1$ in Case I, while it is $k=3$ in Case II. All the subcases identified by an alphabetic symbol "A" satisfy $r_{1} \mu_{1}<r_{2}\left(\mu_{1}+\mu_{2}-k\right)$ which corresponds to Figure 1. Likewise, all the subcases characterized by "B" satisfy $r_{1} \mu_{1} \geq r_{2}\left(\mu_{1}+\mu_{2}-k\right)$ that corresponds to Figure 2. The difference between the two conditions $r_{1} \mu_{1}<r_{2}\left(\mu_{1}+\mu_{2}-k\right)$ and $r_{1} \mu_{1} \geq r_{2}\left(\mu_{1}+\mu_{2}-k\right)$ is such that the former has a larger value of $r_{2} \mu_{2}$ than the latter, and that $r_{1} \mu_{1}-r_{2} \mu_{2}$ under the former condition is small but it is large under the latter condition.

In Table 2 , it is assumed for simplicity that the raw prices $a\left(q_{1}\right)$ and $b\left(q_{2}\right)$ of a system and an expendable, respectively, are constants independently of $q_{1}$ and $q_{2}$, and that the segment size $N_{3}$ of the myopic normal users is set to 1,2 and 3 against $N_{1}=N_{2}=1$. Figure 3 shows three dimensional images of the manufacturer's profit for subcases I-1-A and 
Table 2: Cases

\begin{tabular}{c|c|c|c|c|c|c|c|c|c|c}
\hline Case & $r_{1}$ & $\mu_{1}$ & $r_{2}$ & $\mu_{2}$ & $a\left(q_{1}\right)$ & $b\left(q_{2}\right)$ & $k$ & $N_{1}$ & $N_{2}^{\prime}$ & $N_{3}$ \\
\hline I-1-A & 40 & 20 & 30 & 12 & 50 & 2 & 1 & 1 & 1 & 1 \\
I-1-B & 40 & 20 & 20 & 10 & 50 & 2 & 1 & 1 & 1 & 1 \\
\hline I-2-A & 40 & 20 & 30 & 12 & 50 & 2 & 1 & 1 & 1 & 2 \\
I-2-B & 40 & 20 & 20 & 10 & 50 & 2 & 1 & 1 & 1 & 2 \\
\hline I-3-A & 40 & 20 & 30 & 12 & 50 & 2 & 1 & 1 & 1 & 3 \\
I-3-B & 40 & 20 & 20 & 10 & 50 & 2 & 1 & 1 & 1 & 3 \\
\hline II-1-A & 40 & 20 & 30 & 12 & 50 & 2 & 3 & 1 & 1 & 1 \\
II-1-B & 40 & 20 & 20 & 10 & 50 & 2 & 3 & 1 & 1 & 1 \\
\hline II-2-A & 40 & 20 & 30 & 12 & 50 & 2 & 3 & 1 & 1 & 2 \\
II-2-B & 40 & 20 & 20 & 10 & 50 & 2 & 3 & 1 & 1 & 2 \\
\hline II-3-A & 40 & 20 & 30 & 12 & 50 & 2 & 3 & 1 & 1 & 3 \\
II-3-B & 40 & 20 & 20 & 10 & 50 & 2 & 3 & 1 & 1 & 3 \\
\hline
\end{tabular}

I-1-B, while Figure 4 reveals those for subcases I-3-A and I-3-B. Table 3 summarizes the manufacturer's optimal pricing strategies together with the regions where the manufacturer's globally optimal strategies exist.

Firstly, it can be observed in Figures 3 and 4 that the three dimensional structure of the manufacturer's profit under condition $r_{1} \mu_{1}<r_{2}\left(\mu_{1}+\mu_{2}-k\right)$ is slightly different from that under $r_{1} \mu_{1} \geq r_{2}\left(\mu_{1}+\mu_{2}-k\right)$, which is intuitively imagined from Figures 1 and 2 .

Secondly, let us focus on the subcases discriminated by "A", where $r_{1} \mu_{1}-r_{2} \mu_{2}$ is relatively small. As shown in Table 3 , the optimal strategies in these subcases exist in $\Omega_{3(2)}$, proposing to let the myopic normal users as well as the power users purchase both systems and additional expendables. This is especially insisted in Proposition 1-(ii).

Thirdly, we discuss the subcases characterized by " $\mathrm{B}$ ", where $r_{1} \mu_{1}-r_{2} \mu_{2}$ is not small. It is notable in Table 3 that the optimal strategies in these subcases move from $\Omega_{3(1)}$ to $\Omega_{3(2)}$ as $N_{3}$ increases. This can also be observed in Figures 3-(b) and 4-(b). It is suggested in these subcases that the manufacture should induce the myopic normal users as well as the power users to purchase both systems and expendables particularly by a price markdown from $p_{2}^{*}$ in Equation (4.18) to that in Equation (4.21) on expendables when the segment size of the myopic normal users is large.

\section{Concluding Remarks}

In this paper, we have proposed a theoretical model to discuss optimal pricing strategies for installbase business which deals in a system along with its replaceable parts as expendable supplies under monopoly. The proposed model has introduced three typical types of consumer segments; a segment of power users, a segment of rational normal users and that of myopic normal users. The power users frequently use the system to consume many expendables, while the rational and the myopic normal users do not use the system so frequently and therefore do not consume many expendables. The purchase behaviors of the power users and the rational normal users are rational as postulated in economics in many cases. Those of the myopic normal users are, however, not rational but boundedly rational in the sense that they do not refer to the price of expendables but focus on the system price only when they purchase a system. They will notice the expendable price after they consume all the initially attached expendables. Under these three types of consumer segments, the 


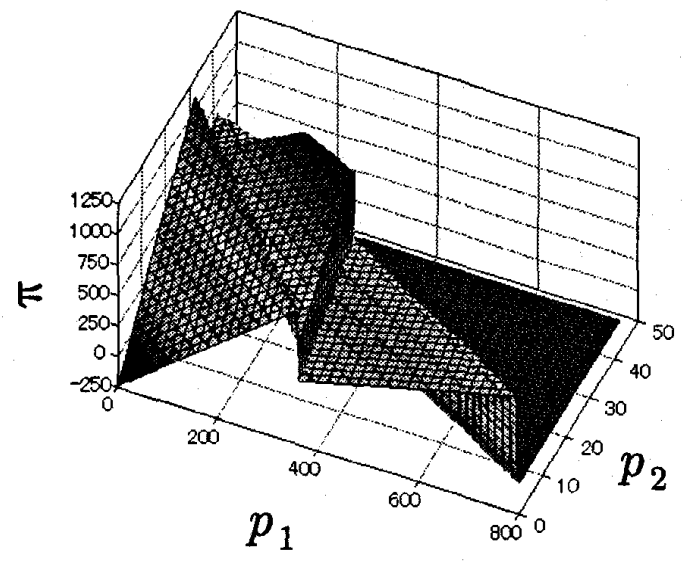

(a) Case I-1-A

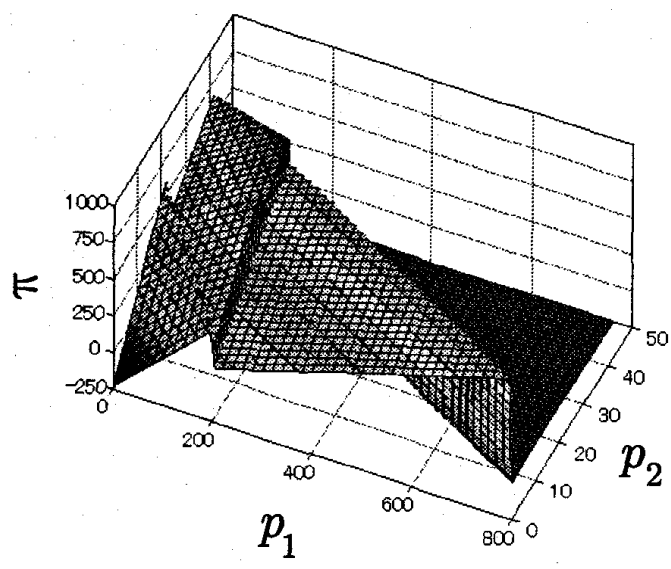

(b) Case I-1-B

Figure 3: Manufacturer's profit (Case I-1)

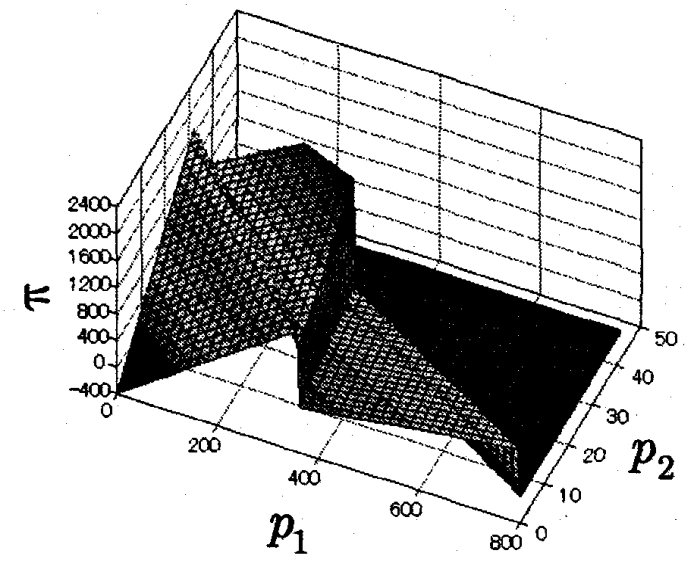

(a) Case I-3-A

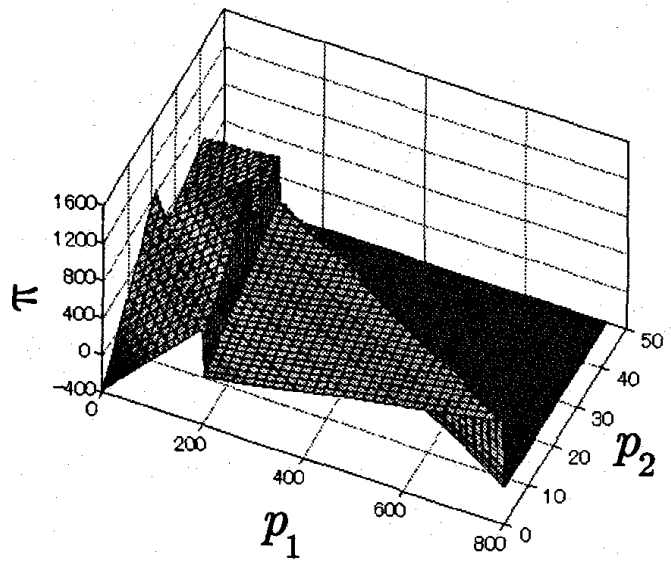

(b) Case I-3-B

Figure 4: Manufacturer's profit (Case I-3) 
Table 3: Optimal Strategy

\begin{tabular}{c|c|r|r|r}
\hline Case & Region & $p_{1}^{*}$ & $p_{2}^{*}$ & $\pi^{*}$ \\
\hline I-1-A & $\Omega_{3(2)}$ & 360 & 23 & 1250 \\
I-1-B & $\Omega_{3(1)}$ & 200 & 32 & 858 \\
\hline I-2-A & $\Omega_{3(2)}$ & 360 & 23 & 1791 \\
I-2-B & $\Omega_{3(2)}$ & 200 & 20 & 1110 \\
\hline I-3-A & $\Omega_{3(2)}$ & 360 & 23 & 2332 \\
I-3-B & $\Omega_{3(2)}$ & 200 & 20 & 1420 \\
\hline II-1-A & $\Omega_{3(2)}$ & 360 & 26 & 1229 \\
II-1-B & $\Omega_{3(1)}$ & 200 & 35 & 854 \\
\hline II-2-A & $\Omega_{3(2)}$ & 360 & 26 & 1748 \\
II-2-B & $\Omega_{3(1)}$ & 200 & 35 & 998 \\
\hline II-3-A & $\Omega_{3(2)}$ & 360 & 26 & 2267 \\
II-3-B & $\Omega_{3(2)}$ & 200 & 20 & 1260 \\
\hline
\end{tabular}

problem has been formulated to consider the manufacturer's optimal pricing strategy for the system and the expendables.

The proposed model has reflected several types of installbase business most of which can be observed in the actual environments. One is the installbase business that targets all the types of consumers. Another type of installbase business ignores the rational normal users to focus on the power users and the myopic normal users, but sets the price of expendables high so that the myopic normal users might not be interested in them even after they consume all the expendables initially attached to each system. The other type of installbase business also targets both the power users and the myopic normal users to sell the additional expendables as well as the systems.

It has been clarified in this study that the manufacturer should never focus on the power users only to make a larger profit but should attract the myopic normal users as well if neither the raw price of the system nor that of the expendable increases with its volume to be produced. In particular, when WTP for the continued system use of the normal user is somewhat analogous to that of the power user, the manufacturer can increase his/her profit by inducing at least the myopic users to purchase both systems and additional expendables. It has also been verified through numerical examples that even if the difference in WTP between the power users and the normal users is not small, the manufacturer should attract the myopic users as well as the power users to use the system continuously by a price markdown of expendables when their segment size is large.

The installbase business should further be studied since

(1) purchase behaviors to more expensive systems might be different from those to cheap ones,

(2) the expendables initially attached to each individual system will play an important role, and therefore the number of those attached expendables is an important factor for the consumers, particularly the myopic normal users, and

(3) when a system expires, the consumer will purchase one again for the repeated use, which is not taken into account in this study.

The authors are grateful to the anonymous reviewers for their useful comments, which have made the manuscript more readable. 


\section{References}

[1] J. Bulow, J.D. Geanakoplos, and P.D. Klemperer: Multimarket oligopoly: Strategic substitutes and complements. Journal of Political Economy, 93 (1985), 488-511.

[2] P. Dubey, O. Haimanko, and A. Zapechelnyuk: Strategic complements and substitutes and potential games. Games and Economic Behavior, 54 (2006), 77-94.

[3] L. Karp, I.H. Lee, and R. Mason: A global game with strategic substitutes and complements. Games and Economic Behavior, 60 (2007), 155-175.

[4] M. Khouja, S.S. Robbins, and H.K. Rajagopalan: Optimal pricing and delayed incentives in a heterogeneous consumer market. Journal of Revenue and Pricing Management, 7 (2008), 85-105.

[5] N.G. Mankiw: Principles of Economics (4th ed.), (Thomson South-Western, Mason, 2007).

[6] H. Sandoh and T. Koide: Optimum pricing in installbase business under monopoly Behavioral economics approach. M.H. Elwany and A.B. Eltawil (eds.): Proceedings of the 37th International Conference on Computers and Industrial Engineering (Alexandria, Egypt, 2007), 367-377.

[7] K.T. Talluri and G.J. van Ryzin: The Theory and Practice of Revenue Management, (Springer-Verlag, New York, 2004).

[8] X. Vives: Nash equilibrium with strategic complementarities. Journal of Mathematical Economics, 19 (1990), 305-321.

[9] C.A. Willson: On the optimum pricing policy of a monopolist. Journal of Political Economy, 96 (1998), 164-176.

\section{A. Proof of Proposition 5.1}

\section{Proof of (i)}

From Equations (4.4) and (4.16), we have

$$
\begin{aligned}
\pi_{3(2) a}^{*}-\pi_{1}^{*}= & N_{1}\left[a\left(N_{1}\right)-a\left(N_{1}+N_{3}\right)+\mu_{1} b\left(N_{1} \mu_{1}\right)-\mu_{1} b\left(N_{1} \mu_{1}+N_{3} k\right)\right] \\
& +N_{3}\left[r_{2} \mu_{2}+\frac{\left(r_{1} \mu_{1}-r_{2} \mu_{2}\right)\left(\mu_{2}-k\right)}{\mu_{1}-k}-a\left(N_{1}+N_{3}\right)-\mu_{2} b\left(N_{1} \mu_{1}+N_{3} \mu_{2}\right)\right],
\end{aligned}
$$

and if $a\left(N_{1}\right) \geq a\left(N_{1}+N_{3}\right)$ and $b\left(N_{1} \mu_{1}\right) \geq b\left(N_{1} \mu_{1}+N_{3} k\right)$, the first term in the right-handside of the above equation becomes non-negative. In addition, Assumption (10) indicates the second term in the right-hand-side is positive, and hence we have

$$
\pi_{3(2) a}^{*}-\pi_{1}^{*}>0
$$

\section{Proof of (ii)}

Equations (4.12) and (4.16) yield

$$
\begin{aligned}
\pi_{3(2) a}^{*}-\pi_{3(1) a}^{*}= & N_{1} \mu_{1}\left[b\left(N_{1} \mu_{1}+N_{3} k\right)-b\left(N_{1} \mu_{1}+N_{3} \mu_{2}\right)\right] \\
& +N_{3}\left[r_{2} \mu_{2}-r_{1} \mu_{1}+\frac{\left(r_{1} \mu_{1}-r_{2} \mu_{2}\right)\left(\mu_{2}-k\right)}{\mu_{1}-k}+r_{2}\left(\mu_{1}-k\right)\right. \\
& \left.+k b\left(N_{1} \mu_{1}+N_{3} k\right)-\mu_{2} b\left(N_{1} \mu_{1}+N_{3} \mu_{2}\right)\right]
\end{aligned}
$$


If $b\left(N_{1} \mu_{1}+N_{3} k\right) \geq b\left(N_{1} \mu_{1}+N_{3} \mu_{2}\right)$, we have

$$
\begin{aligned}
\pi_{3(2) a}^{*}-\pi_{3(1) a}^{*} \geq & N_{1} \mu_{1}\left[b\left(N_{1} \mu_{1}+N_{3} k\right)-b\left(N_{1} \mu_{1}+N_{3} \mu_{2}\right)\right] \\
& +N_{3}\left[r_{2} \mu_{2}-r_{1} \mu_{1}+\frac{\left(r_{1} \mu_{1}-r_{2} \mu_{2}\right)\left(\mu_{2}-k\right)}{\mu_{1}-k}+r_{2}\left(\mu_{1}-k\right)\right. \\
& \left.-\left(\mu_{2}-k\right) b\left(N_{1} \mu_{1}+N_{3} \mu_{2}\right)\right]
\end{aligned}
$$

Let $A$ be given by

$$
A \equiv r_{2} \mu_{2}-r_{1} \mu_{1}+\frac{\left(r_{1} \mu_{1}-r_{2} \mu_{2}\right)\left(\mu_{2}-k\right)}{\mu_{1}-k}+r_{2}\left(\mu_{1}-k\right)-\left(\mu_{2}-k\right) b\left(N_{1} \mu_{1}+N_{3} \mu_{2}\right)
$$

and then $A$ satisfies

$$
\begin{aligned}
A & >r_{2} \mu_{2}-r_{1} \mu_{1}+r_{2}\left(\mu_{1}-k\right)+\frac{\mu_{2}-k}{\mu_{1}-k}\left[r_{1} \mu_{1}-r_{2} \mu_{2}-r_{2}\left(\mu_{1}-k\right)\right] \\
& =\left[r_{2}\left(\mu_{1}+\mu_{2}-k\right)-r_{1} \mu_{1}\right]\left(1-\frac{\mu_{2}-k}{\mu_{1}-k}\right)>0
\end{aligned}
$$

which agrees with

$$
\pi_{3(2) a}^{*}-\pi_{3(1) a}^{*}>0
$$

since we have $r_{2}>b\left(N_{1} \mu_{1}+N_{3} \mu_{2}\right)$ from Assumption (10).

\section{B. Proof of Proposition $\mathbf{5 . 2}$}

When $a\left(N_{1}\right) \geq a\left(N_{1}+N_{3}\right)$ and $b\left(N_{1} \mu_{1}\right) \geq b\left(N_{1} \mu_{1}+N_{3} k\right)$, we have

$$
\begin{aligned}
\pi_{3(1) b}^{*}-\pi_{1}^{*}= & N_{1}\left[a\left(N_{1}\right)-a\left(N_{1}+N_{3}\right)+\mu_{1} b\left(N_{1} \mu_{1}\right)-\mu_{1} b\left(N_{1} \mu_{1}+N_{3} k\right)\right] \\
& +N_{3}\left[r_{2} \mu_{2}-a\left(N_{1}+N_{3}\right)-k b\left(N_{1} \mu_{1}+N_{3} k\right)\right]>0
\end{aligned}
$$

since Assumption (10) yields $r_{2} \mu_{2}-a\left(N_{1}+N_{3}\right)-k b\left(N_{1} \mu_{1}+N_{3} k\right)>0$.

Hiroaki Sandoh

Graduate School of Economics

Osaka University

1-7, Machikaneyama-cho, Toyonaka

Osaka 560-0043, Japan

E-mail: sandoh@econ.osaka-u.ac.jp 